\title{
Gamma-ray bursts: optical afterglows in the deep Newtonian phase
}

\author{
Y. F. Huang ${ }^{1,2,3 \star}$ and K. S. Cheng ${ }^{2} \star$ \\ ${ }^{1}$ Department of Astronomy, Nanjing University, Nanjing 210093, China \\ ${ }^{2}$ Department of Physics, The University of Hong Kong, Pokfulam Road, Hong Kong, China \\ ${ }^{3}$ LCRHEA, Institute for High-Energy Physics, Chinese Academy of Sciences, Beijing 100039, China
}

MNRAS in press (originally submitted in October, 2002)

\begin{abstract}
Gamma-ray burst remnants become trans-relativistic typically in days to tens of days, and they enter the deep Newtonian phase in tens of days to months, during which the majority of shock-accelerated electrons will no longer be highly relativistic. However, a small portion of electrons are still accelerated to ultra-relativistic speeds and capable of emitting synchrotron radiation. The distribution function for electrons is re-derived here so that synchrotron emission from these relativistic electrons can be calculated. Based on the revised model, optical afterglows from both isotropic fireballs and highly collimated jets are studied numerically, and compared to analytical results. In the beamed cases, it is found that, in addition to the steepening due to the edge effect and the lateral expansion effect, the light curves are universally characterized by a flattening during the deep Newtonian phase.
\end{abstract}

Key words: radiation mechanisms: non-thermal - stars: neutron - ISM: jets and outflows - gamma-rays: bursts

\section{INTRODUCTION}

Observations of X-ray, optical and radio afterglows from gamma-ray bursts (GRBs) definitely show that at least most long GRBs are of cosmological origin (e.g. Costa et al. 1997; Frail et al. 1997; Galama et al. 1997; Metzger et al. 1997; Kulkarni et al. 1998; Garcia et al. 1998; Piro et al. 1998; Pedersen et al. 1998; Galama et al. 1998a,b; Akerlof et al. 1999; Vreeswijk et al. 1999; Zhu et al. 1999; Hjorth et al. 2002). The famous fireball model, which incorporates internal shocks to account for the main bursts, and external shocks to account for afterglows, becomes the most popular model (Mészáros \& Rees 1992; Rees \& Mészáros 1992; Mészáros, Laguna \& Rees 1993; Mészáros, Rees \& Papathanassiou 1994; Katz 1994; Rees \& Mészáros 1994; Sari, Narayan \& Piran 1996). Observed features of GRB afterglows can basically be well explained in this frame-work (Mészáros \& Rees 1997; Vietri 1997; Waxman 1997a,b; Wijers, Rees \& Mészáros 1997; Mészáros, Rees \& Wijers 1998; Sari, Piran \& Narayan 1998; Dai \& Lu 1998, 1999; Dermer, Böttcher \& Chiang 1999a; Dermer, Chiang \& Böttcher 1999b; Wijers \& Galama 1999; Wijers et al. 1999). However, we are still far from resolving the puzzle of GRBs, since many crucial information concerning the true nature of the inner engine is lost after an initial acceleration phase

\footnotetext{
* E-mail: hyf@nju.edu.cn(YFH); hrspksc@hkucc.hku.hk(KSC)
}

of the fireball evolution. Studies on afterglows can hopefully provide important clues of GRB progenitors. For example, from afterglow observations, we can measure the beaming angle, the environment density, the total kinetic energy etc (Hjorth et al. 2002; Königl \& Granot 2002). For recent reviews on GRBs, see Piran (1999), van Paradijs, Kouveliotou \& Wijers (2000) and Mészáros (2002).

As the most violent bursts in the Universe since the Big Bang, GRBs are most impressing for their extremely relativistic motions, with Lorentz factor $\gamma \geq 100-1000$. In 1997, Wijers et al. (1997) discussed the non-relativistic phase of GRB afterglows for the first time. But at the beginning of the afterglow era, the Newtonian aspects of GRBs were largely ignored in the literature for obvious reasons. The importance of non-relativistic phase was stressed and then extensively discussed by Huang et al. (Huang et al. 1998a; Huang, Dai \& Lu 1998b, 1999a,b; Huang et al. 2000a; Huang, Dai \& Lu 2000b,c), who pointed out that GRB remnants enter the trans-relativistic phase (here we define it as $2 \leq \gamma \leq 5$ ) typically in a few to 10 days. Today, the importance of Newtonian phase has been realized by more and more authors (e.g. Kobayashi, Piran \& Sari 1999; Livio \& Waxman 2000; Chevalier \& Li 2000; Frail, Waxman \& Kulkarni 2000; Weiler et al. 2002; Kulkarni 2002).

Afterglows from both isotropic fireballs and highly collimated jets in the trans-relativistic and non-relativistic phases have been investigated in great detail by Huang et 
al. (1998b, 1999a,b, 2000a,b,c). Although their dynamical equations are valid for sufficiently long period, their discussion cannot extend to the deep Newtonian phase, during which most shock-accelerated electrons will cease to be ultra-relativistic and will no longer emit synchrotron radiation. In this article, a more reasonable distribution function will be derived for electrons, so that we can extend our calculation into the deep Newtonian phase. The structure of our article is arranged as follows. We describe our dynamical and radiation model in Section 2. Based on the revised model, optical afterglows from isotropic fireballs, conical jets as well as cylindrical jets are then investigated numerically in Section 3. Emphases will be put on the light curve behaviour in the deep Newtonian phase. Section 4 is a brief discussion.

\section{MODEL}

The overall dynamical evolution of GRB remnants has been studied by many authors (Huang et al. 1999a,b; Kobayashi et al. 1999; Dermer \& Humi 2001; Panaitescu \& Kumar 2001a, 2002). Here we adopt the simple dynamical model proposed by Huang et al. (1999a,b), which is characterized mainly by the following differential equation,

$\frac{d \gamma}{d m}=-\frac{\gamma^{2}-1}{M_{\mathrm{ej}}+\epsilon m+2(1-\epsilon) \gamma m}$,

where $\gamma$ is the bulk Lorentz factor of the shocked interstellar medium (ISM), $M_{\mathrm{ej}}$ is the initial ejecta mass, $\epsilon$ is the radiative efficiency and $m$ is the swept-up ISM mass. It has been shown that this equation is correct for both ultra-relativistic and non-relativistic blastwaves, no matter whether they are adiabatic or highly radiative (Huang et al. 1999a,b). In realistic GRB remnants, the blastwaves can be radiative only within the initial two or three hours following the main burst (Dai, Huang \& Lu 1999). They will become completely adiabatic after that period. So, for simplicity, we will take $\epsilon \equiv 0$ directly in this study.

For beamed ejecta with a half opening angle of $\theta$, Huang et al. $(2000 \mathrm{a}, \mathrm{b})$ have proposed an improved method to describe the lateral expansion of the remnant. Their description is based on a refined expression for the sound speed that is appropriate in both relativistic and non-relativistic phases. In Section 3, we will solve the dynamical evolution of isotropic fireballs, conical jets as well as cylindrical jets numerically. For details of the numerical procedure, we refer readers to Huang et al. (1999b, 2000a,b) and Cheng, Huang \& Lu (2001).

Synchrotron radiation from the shock-accelerated ISM electrons plays a major role in the optical afterglows. To calculate this emission, two factors are essential to be known: the magnetic field and the energy of electrons. As usual, we assume that the magnetic energy density in the comoving frame is a fraction $\xi_{\mathrm{B}}^{2}$ of the total thermal energy density $\left(B^{\prime 2} / 8 \pi=\xi_{\mathrm{B}}^{2} e^{\prime}\right)$. Electrons are also usually assumed to carry a fraction $\xi_{\mathrm{e}}$ of the proton energy and follow a power-law distribution according to their Lorentz factors,

$\frac{d N_{\mathrm{e}}^{\prime}}{d \gamma_{\mathrm{e}}} \propto \gamma_{\mathrm{e}}^{-p}, \quad\left(\gamma_{\mathrm{e}, \min } \leq \gamma_{\mathrm{e}} \leq \gamma_{\mathrm{e}, \max }\right)$

where the minimum Lorentz factor can be easily derived as, $\gamma_{\mathrm{e}, \min }=\xi_{\mathrm{e}} \frac{m_{\mathrm{p}}}{m_{\mathrm{e}}} \cdot \frac{p-2}{p-1}(\gamma-1)+1$

with $m_{\mathrm{p}}$ and $m_{\mathrm{e}}$ being the proton and electron mass respectively. The maximum Lorentz factor is constrained by synchrotron cooling, $\gamma_{\mathrm{e}, \max } \approx 10^{8}\left(B^{\prime} / 1 \mathrm{G}\right)^{-1 / 2}$ (Mészáros et al. 1993; Vietri 1997; Totani 1999). Sari et al. (1998) further suggested that radiation loss may play an important role in the process and can change the distribution function to $d N_{\mathrm{e}}^{\prime} / d \gamma_{\mathrm{e}} \propto \gamma_{\mathrm{e}}^{-(p+1)}$ for electrons above a critical Lorentz factor $\gamma_{\mathrm{c}}$, which is given by $\gamma_{\mathrm{c}}=6 \pi m_{\mathrm{e}} c /\left(\sigma_{\mathrm{T}} \gamma B^{\prime 2} t\right)$, where $\sigma_{\mathrm{T}}$ is the Thomson cross section and $t$ is the time of the observer. Based on these considerations, a detailed description of electron distribution has been presented by Dai et al. (1999, also see Huang et al. 2000a,b).

The above discussion is valid only when electrons are ultra-relativistic, i.e., $\gamma_{e, \text { min }} \gg 1$. However, in the deep Newtonian phase, this condition will no longer be satisfied. The bulk Lorentz factor $(\gamma)$ will be so close to unity that $\gamma_{\mathrm{e}, \mathrm{min}}$ will also be very close to one. It means the majority of electrons will cease to be ultra-relativistic. For example, taking typical values of $\xi_{\mathrm{e}}=0.1$ and $p=2.2, \gamma_{\mathrm{e} \text {,min }}$ will equal to 5 when $\gamma \approx 1.13$ (or $\beta=\sqrt{\gamma^{2}-1} / \gamma \approx 0.47$ ). Anyway, the distribution of electrons with respect to their kinetic energies can still be assumed to be a power-law function, which now takes the following form,

$\frac{d N_{\mathrm{e}}^{\prime}}{d \gamma_{\mathrm{e}}} \propto\left(\gamma_{\mathrm{e}}-1\right)^{-p}, \quad\left(\gamma_{\mathrm{e}, \min } \leq \gamma_{\mathrm{e}} \leq \gamma_{\mathrm{e}, \max }\right)$.

Most electrons are now non-relativistic and their cyclotron radiation cannot be observed in optical bands. But there are still many relativistic electrons with Lorentz factor above a critical value, $\gamma_{e, s y n}$, and still capable of emitting synchrotron radiation. In this case, to calculate the optical afterglow, we just need to integrate the emission over all those electrons satisfying $\gamma_{\mathrm{e}, \mathrm{syn}} \leq \gamma_{\mathrm{e}} \leq \gamma_{\mathrm{e} \text {,max }}$. The determination of $\gamma_{\mathrm{e}, \mathrm{syn}}$ is somewhat arbitrary. We believe that it could be some value like 5 or 10 . Anyway, it is lucky that this uncertainty will not bring any essential effect into the optical afterglows (see Section 3).

Based on equation (4), we can now re-derive the electron distribution as follows, following Dai et al.'s (1999) treatment (also see Huang et al. 2000a,b),

(i) For $\gamma_{\mathrm{c}} \leq \gamma_{\mathrm{e}, \mathrm{min}}$,

$\frac{d N_{\mathrm{e}}^{\prime}}{d \gamma_{\mathrm{e}}}=C_{1}\left(\gamma_{\mathrm{e}}-1\right)^{-(p+1)}, \quad\left(\gamma_{\mathrm{e}, \min } \leq \gamma_{\mathrm{e}} \leq \gamma_{\mathrm{e}, \max }\right)$,

$C_{1}=\frac{p}{\left(\gamma_{\mathrm{e}, \min }-1\right)^{-p}-\left(\gamma_{\mathrm{e}, \max }-1\right)^{-p}} N_{\mathrm{ele}}$,

where $N_{\text {ele }}$ is the total number of radiating electrons involved.

(ii) For $\gamma_{\mathrm{e}, \min }<\gamma_{\mathrm{c}} \leq \gamma_{\mathrm{e}, \max }$,

$\frac{d N_{\mathrm{e}}^{\prime}}{d \gamma_{\mathrm{e}}}= \begin{cases}C_{2}\left(\gamma_{\mathrm{e}}-1\right)^{-p}, & \left(\gamma_{\mathrm{e}, \min } \leq \gamma_{\mathrm{e}} \leq \gamma_{\mathrm{c}}\right), \\ C_{3}\left(\gamma_{\mathrm{e}}-1\right)^{-(p+1)}, & \left(\gamma_{\mathrm{c}}<\gamma_{\mathrm{e}} \leq \gamma_{\mathrm{e}, \max }\right),\end{cases}$

where

$C_{2}=C_{3} /\left(\gamma_{\mathrm{c}}-1\right)$,

$$
C_{3}=\left[\frac{\left(\gamma_{\mathrm{e}, \min }-1\right)^{1-p}-\left(\gamma_{\mathrm{c}}-1\right)^{1-p}}{(p-1)\left(\gamma_{\mathrm{c}}-1\right)}\right.
$$


$\left.+\frac{\left(\gamma_{\mathrm{c}}-1\right)^{-p}-\left(\gamma_{\mathrm{e}, \max }-1\right)^{-p}}{p}\right]^{-1} N_{\text {ele }}$.

(iii) If $\gamma_{\mathrm{c}}>\gamma_{\mathrm{e}, \max }$, then

$\frac{d N_{\mathrm{e}}^{\prime}}{d \gamma_{\mathrm{e}}}=C_{4}\left(\gamma_{\mathrm{e}}-1\right)^{-p}, \quad\left(\gamma_{\mathrm{e}, \min } \leq \gamma_{\mathrm{e}} \leq \gamma_{\mathrm{e}, \max }\right)$,

where

$C_{4}=\frac{p-1}{\left(\gamma_{\mathrm{e}, \min }-1\right)^{1-p}-\left(\gamma_{\mathrm{e}, \max }-1\right)^{1-p}} N_{\text {ele }}$.

Of course, $\gamma_{\mathrm{c}} \gg 1$ and $\gamma_{\mathrm{e}, \max } \gg 1$ are safely satisfied, so that $\gamma_{\mathrm{c}}-1$ and $\gamma_{\mathrm{e}, \max }-1$ can be simplified as $\gamma_{\mathrm{c}}$ and $\gamma_{\mathrm{e}, \max }$ respectively in these equations.

\section{NUMERICAL RESULTS}

Afterglows from isotropic fireballs as well as collimated jets have been studied in great detail by Huang et al. (Huang et al. 1999a,b, 2000a,b,c; Cheng, Huang \& Lu 2001). However, those discussion in general can not extend to the deep Newtonian phase. In this section, we use our revised model to continue the study. In all our calculations here, we take the following typical parameter values: $\gamma_{\mathrm{e}, \mathrm{syn}}=5, \xi_{\mathrm{e}}=$ $0.1, D_{\mathrm{L}}=1 \mathrm{Gpc}$ and $p=2.2$, where $D_{\mathrm{L}}$ is the luminosity distance. The initial value of $\gamma$ is fixedly taken as $\gamma_{0}=300$. Other parameters, such as the total isotropic kinetic energy of the blastwave $\left(E_{0}\right)$, the number density of the interstellar medium $(n)$ and the magnetic energy fraction $\left(\xi_{\mathrm{B}}^{2}\right)$, will be given separately elsewhere. The effects of equal arrival time surfaces (Waxman 1997c; Sari 1998; Panaitescu \& Mészáros 1998; Huang et al. 2000a,b) are taken into account in our studies. For more details of the calculation, readers are refered to Huang et al. (1999b, 2000a,b) and Cheng, Huang \& $\mathrm{Lu}(2001)$.

\subsection{Isotropic fireballs}

Fig. 1 shows the evolution of the Lorentz factor for some exemplary isotropic fireballs and the corresponding $R$-band flux density $\left(S_{\mathrm{R}}\right)$. Analytical solution requires that the fireball evolves as $\gamma \propto t^{-3 / 8}$ in the ultra-relativistic phase and $\beta=\sqrt{\gamma^{2}-1} / \gamma \propto t^{-3 / 5}$ in the Newtonian limit (Wijers et al. 1997; Huang et al. 1999a,b). Fig. 1a consists with the requirement exactly. Wijers et al. (1997) and Dai \& Lu (1999, 2000) have also derived the theoretical afterglow light curve analytically for both relativistic and non-relativistic phases. In case of slow cooling, we have

$S_{\mathrm{R}} \propto \begin{cases}t^{(3-3 p) / 4}, & (\gamma \gg 1), \\ t^{(21-15 p) / 10}, & (\beta \ll 1) .\end{cases}$

Taking $p=2.2$, we get $S_{\mathrm{R}} \propto t^{-0.9}$ and $S_{\mathrm{R}} \propto t^{-1.2}$ for relativistic and Newtonian phases respectively. Our numerical results are consistent with their solutions. For example, the slope of the solid line in Fig. $1 \mathrm{~b}$ is $\sim-0.92$ and $\sim-1.22$ in the relativistic phase and the non-relativistic phase respectively.

Fig. 1 also clearly shows that the remnant enters the deep Newtonian phase in a relatively short period. For example, $\gamma_{\mathrm{e}, \mathrm{min}}$ becomes less than $\gamma_{\mathrm{e}, \mathrm{syn}}$ at $\sim 10^{7} \mathrm{~s}$, i.e., about 3 months. Since optical afterglows from some GRBs have been detected for more than six months, and radio afterglows are even detectable one or three years later (Frail et al. 2000; Kulkarni 2002), we see that the study of afterglows in the deep Newtonian phase is really essential.

In our calculations, we have assumed that the minimum Lorentz factor of electrons capable of emitting synchrotron radiation is $\gamma_{\mathrm{e}, \mathrm{syn}}=5$. This evaluation is somewhat arbitrary, but it does not affect the optical light curve too much. The characteristic synchrotron frequency of an electron with Lorentz factor $\gamma_{\mathrm{e}}$ in a magnetic field $B^{\prime}$ is $\nu=\gamma_{\mathrm{e}}^{2} e B^{\prime} /\left(2 \pi m_{\mathrm{e}} c\right) \approx 2.8 \times 10^{6} \gamma_{\mathrm{e}}^{2}\left(B^{\prime} / 1 \mathrm{G}\right) \mathrm{Hz}$. Electrons in the lower energy range thus do not contribute to optical flux density significantly. In fact, we have taken $\gamma_{\mathrm{e}, \mathrm{syn}}$ as 10 or even 50 in our trial calculations and just found no difference in the optical light curves.

\subsection{Conical jets}

Collimation is very important in GRBs, which can affect the intrinsic kinetic energy and may provide direct clues on the progenitors (Frail et al. 2001; Ioka \& Nakamura 2001; Panaitescu \& Kumar 2001a,b, 2002; Ramirez-Ruiz \& Lloyd-Ronning 2002; Yamazaki, Ioka \& Nakamura 2002). Beaming effects in afterglows have been discussed extensively in the literature. It is generally believed that due to both the edge effect (Kulkarni et al. 1999; Mészáros \& Rees 1999; Panaitescu \& Mészáros 1999) and the lateral expansion effect (Rhoads 1997, 1999), afterglows from a conical jet are characterized by a break in the light curve. The break point is approximately determined by $\gamma \sim 1 / \theta_{0}$, where $\theta_{0}$ is the initial half opening angle. GRBs 990123, 990510 and 000301c are regarded as good examples (Castro-Tirado et al. 1999; Harrison et al. 1999; Kulkarni et al. 1999; Sari, Piran \& Halpern 1999; Wijers et al. 1999; Masetti et al. 2000; Holland et al. 2000; Berger et al. 2000; Jensen et al. 2001; Huang et al. 2000c). However, detailed numerical studies show that the break is usually quite smooth (Moderski, Sikora \& Bulik 2000). Huang et al. further found that the break is parameter-dependent (Huang et al. 2000a,b). They also suggested that the trans-relativistic and non-relativistic stages are important and should be considered carefully. In this section, we use our revised model to reveal the behaviour of conical jets in the deep Newtonian phase. For more details of the calculation, please refer to Huang et al. (2000a,b).

\subsubsection{Conical jets without lateral expansion}

Fig. 2 illustrates the evolution and afterglows of some exemplary conical jets without lateral expansion. In fact, the dynamical evolution of such a jet should be very similar to that of an isotropic fireball, i.e., $\gamma \propto t^{-3 / 8}$ in the ultra-relativistic phase and $\beta \propto t^{-3 / 5}$ in the Newtonian stage. Fig. 2a shows these trends exactly. As for the optical light curve, analytical solutions predict that it should follow $S_{\mathrm{R}} \propto t^{(3-3 p) / 4}$ $\left(t^{-0.9}\right.$ for $\left.p=2.2\right)$ before $\gamma=1 / \theta_{0}=10$. But soon after the $\gamma=10$ point, a break should appear and the light curve becomes $S_{\mathrm{R}} \propto t^{-3 p / 4}\left(t^{-1.65}\right.$ for $\left.p=2.2\right)$. This relation can be derived as follows. Analytically we have $\gamma \propto t^{-3 / 8}$ and $R \propto t^{1 / 4}$. Then the characteristic synchrotron frequency is $\nu_{\mathrm{m}} \propto \gamma \gamma_{\mathrm{e}, \min }^{2} B^{\prime} \propto \gamma^{4} \propto t^{-3 / 2}$, and the corresponding maximum flux density is $S_{\nu, \max } \propto N_{\text {ele }} \gamma B^{\prime} / \gamma^{-2} \propto R^{3} \gamma^{4} \propto t^{-3 / 4}$, 
where the $\gamma^{-2}$ item in $S_{\nu, \max }$ arises from the edge effect when $\gamma<1 / \theta_{0}$. So, the observed optical flux density is $S_{\mathrm{R}} \approx S_{\nu, \max }\left(\nu_{\mathrm{R}} / \nu_{\mathrm{m}}\right)^{-(p-1) / 2} \propto t^{-3 p / 4}$. Such a light curve break can be clearly seen in Fig. 2b. For example, the solid line can be fit as $S_{\mathrm{R}} \propto t^{-0.89}$ during $10^{3}-10^{4} \mathrm{~s}$ and it is approximately $S_{\mathrm{R}} \propto t^{-1.80}$ during $10^{5}-10^{6} \mathrm{~s}$. The slopes are acceptably consistent with analytic values. However, it should be noted that the break occurs obviously later than the time determined by $\gamma=1 / \theta_{0}=10$, which has been pointed out by Huang et al. in previous studies (Huang et al. 2000a,b).

Maybe the most striking feature in Fig. $2 \mathrm{~b}$ is the flattening of the light curves in the deep Newtonian phase. This is not difficult to understand. In such a non-relativistic phase, the remnant, although beamed, should behaves more or less like a spherical shell since the edge effect no longer exists (Livio \& Waxman 2000). Beaming will only reduce the total flux density by a constant factor. In fact, the slope of the solid line is approximately -1.2 after $10^{9} \mathrm{~s}$, which is just the value expected for an isotropic fireball (see Section 3.1). So, we see that the overall evolution of optical afterglows from a conical jet without lateral expansion can be typically described by,

$$
S_{\mathrm{R}} \propto \begin{cases}t^{(3-3 p) / 4}, & \left(\gamma>1 / \theta_{0}\right), \\ t^{-3 p / 4}, & \left(\gamma<1 / \theta_{0} \text { and } \beta \sim 1\right), \\ t^{(21-15 p) / 10}, & (\beta \ll 1) .\end{cases}
$$

\subsubsection{Conical jets with lateral expansion}

In realistic cases, jets may expand laterally at comoving sound speed. Fig. 3a shows the evolution of Lorentz factor for such realistic conical jets. The curves can be approximately fit by $\gamma \propto t^{-0.39}$ in the highly relativistic phase and by $\gamma-1 \propto t^{-1.25}$ in the non-relativistic phase, consistent with the analytic results of $\gamma \propto t^{-3 / 4}$ and $\gamma-1 \propto t^{-6 / 5}$ respectively. Again we see that $\gamma_{\mathrm{e}, \text { min }}$ becomes less than 5 when $t \geq 3 \times 10^{6}-2 \times 10^{7} \mathrm{~s}$.

Fig. $3 \mathrm{~b}$ illustrates the $R$-band afterglows correspondingly. We see that the general behaviour of the light curves is very similar to that in Fig. 2b. However, slight difference can still be found and deserves addressing in some detail. For example, the solid line approximately follows $S_{\mathrm{R}} \propto t^{-1.03}$ in the ultra-relativistic phase, which is steeper by $\sim t^{0.14}$ than that in Fig. 2b. It indicates that the lateral expansion tends to make the afterglow decay faster. Subsequently the light curve steepens to $S_{\mathrm{R}} \propto t^{-2.24}$, which is also steeper by $\sim t^{0.44}$ than that in Fig. 2b. It is clear that lateral expansion contributes to the light curve break significantly. In the deep Newtonian phase, the light curves are again markedly characterized by a flattening. The slope of the solid line in this segment is $\sim-1.26$, also consistent with the analytical result of -1.2 for an isotropic fireball. In short, we conclude that the overall evolution of optical afterglows from a conical jet with lateral expansion can be typically expressed as,

$$
S_{\mathrm{R}} \propto \begin{cases}t^{(3-3 p) / 4}, & \left(\gamma>1 / \theta_{0}\right), \\ t^{-p}, & \left(\gamma<1 / \theta_{0} \text { and } \beta \sim 1\right), \\ t^{(21-15 p) / 10}, & (\beta \ll 1) .\end{cases}
$$

\subsection{Cylindrical jets}

The geometry of GRB jets is usually assumed to be conical. However, many of the relativistic outflows in radio galaxies are found to maintain constant cross-sections at large scales (Perley, Bridle \& Willis 1984; Biretta, Sparks \& Macchetto 1999). This has led Cheng et al. (2001) to suggest that GRB jets might also be cylindrical. In fact, a very similar idea, i.e., the so called cannon-ball model, has been suggested as the GRB trigger mechanism by Dar et al. (Shaviv \& Dar 1995; Dar 1998), and observed afterglows have also been examined carefully in this frame (Dado, Dar \& De Rújula 2002).

Theoretical GRB afterglows from cylindrical jets have been studied by Cheng et al. (2001) in great detail. However, their discussion again cannot extend to the deep Newtonian phase. Here we use our revised model to continue the study. Since a cylindrical jet without lateral expansion generally decelerates very slowly and can remain to be highly relativistic for as long as $\sim 10^{8}-10^{9} \mathrm{~s}$ (Cheng et al. 2001), we will discuss only cylindrical jets with lateral expansion.

Fig. 4a shows the evolution of $\gamma$ for some exemplary cylindrical jets. In the highly relativistic phase the curves approximately follow $\gamma \propto t^{-0.5}$, and in the non-relativistic phase they follow $\gamma-1 \propto t^{-1.17}$. The timing indices are consistent with the analytical results of $-1 / 2$ and $-6 / 5$ respectively (Cheng et al. 2001). Note that in this figure, $\gamma_{\mathrm{e}, \text { min }}$ is already less than 5 after $\sim(2-4) \times 10^{6} \mathrm{~s}$. Fig. $4 \mathrm{~b}$ illustrates the evolution of optical afterglows correspondingly. In the ultra-relativistic phase, the solid line can be approximated as $S_{\mathrm{R}} \propto t^{-2.34}$, consisting with the analytical solution of $S_{\mathrm{R}} \propto t^{-p}$ (Cheng et al. 2001). After entering the non-relativistic phase, the remnant is expected to resemble an isotropic fireball, so that the light curve should be $S_{\mathrm{R}} \propto t^{(21-15 p) / 10}$ (Cheng et al. 2001). Taking $p=2.2$, we get the analytical timing index of -1.2 . In fact, such a flattening is really observed in Fig. 4b, and the slope of the solid line is just $\sim-1.33$ in the deep Newtonian phase. From these studies, we see that the optical afterglow from laterally expanding cylindrical jets can typically be well represented by,

$S_{\mathrm{R}} \propto \begin{cases}t^{-p}, & (\gamma \gg 1), \\ t^{(21-15 p) / 10}, & (\beta \ll 1) .\end{cases}$

\section{DISCUSSION AND CONCLUSIONS}

We have shown that typically in $\sim 10^{7} \mathrm{~s}$, the majority of shock-accelerated electrons will become non-relativistic, i.e., $\gamma_{\mathrm{e}, \mathrm{min}} \leq \gamma_{\mathrm{e}, \mathrm{syn}}$. These electrons can only emit cyclotron radiation which falls mainly in the frequency range of $\sim 10^{6}$ $-10^{7} \mathrm{~Hz}$. However, a small portion of electrons are still highly relativistic and capable of emitting synchrotron radiation. A revised distribution function has been derived for electrons, which allows us to calculate the emission from these relativistic particles.

Optical afterglows from both isotropic fireballs and highly collimated jets are studied numerically based on the revised model. For isotropic fireballs, the optical light curve steepens by $t^{(15 p-27) / 20}$ after entering the deep Newtonian phase. This corresponds to $\sim t^{0.3}$ for $p=2.2$, or $\sim t^{0.9}$ for $p=3$. For conical jets, we find that the light curve steepens slightly later than the moment determined by $\gamma=1 / \theta_{0}$. It 
is clear that this steepening is due to the edge effect and is strengthened by lateral expansion. Additionally, the optical light curves are universally characterized by a striking flattening during the deep Newtonian phase, which can be attributed to the resembling of the remnant to an isotropic fireball at such late stages (Livio \& Waxman 2000). The flattening is also present in the light curves of cylindrical jets.

Long-lasting optical afterglows have been observed from a number of GRBs, e.g. GRBs 970228, 970508, 991208 (Fruchter et al. 1999, 2000; Castro-Tirado et al. 2001). Optical transients in these events are typically detectable for $\sim 100-200 \mathrm{~d}$. Our studies here are of obvious importance in such cases. However, the flattening of the light curve in the deep Newtonian phase predicted for highly collimated jets has not been observed in realistic observations of jet candidates. A possible reason may be that the optical flux densities are usually too faint. In fact, to observe this flattening clearly, we should follow the optical transient for at least $\sim 10^{8}$ s, i.e., more than $\sim 3$ years. This could hardly be actualized even with the Keck telescope and the Hubble Space Telescope. Additionally, optical emission from the host galaxy makes the measurement even more difficult. Anyway, the next generation optical telescopes may cast some light on the problem.

Radio afterglows can hopefully be observed for even longer period. In fact, a 450-day light curve of the radio afterglow of GRB 970508 has been reported by Frail et al. (2000). GRB 980703 has even been monitored in radio bands for more than 1000 days (Kulkarni 2002). At such stages, the GRB remnant should have entered the deep Newtonian phase. Observations of radio afterglows on such a late stage have the priority that they allow for a direct measure of the intrinsic kinetic energy (Frail et al. 2000). The effect of $\gamma_{\mathrm{e}, \mathrm{min}}$ will have to be considered in fitting those radio light curves theoretically. Calculation of radio emission involves synchrotron self absorption, which is beyond the scope of this article and will be discussed elsewhere.

Orphan afterglow survey is thought to be a useful method to determine the degree of beaming in GRBs (Rhoads 1997; Mészáros, Rees \& Wijers 1999; Totani \& Panaitescu 2002; Nakar, Piran \& Granot 2002; Granot et al. 2002). However, the method is also troubled by many problems (Dalal, Griest \& Pruet 2002; Huang, Dai \& Lu 2002; Levinson et al. 2002; Nakar \& Piran 2003). For example, as suggested by Huang et al. (2002), the method is greatly complicated by the possibility that there might be many failed GRBs, i.e., isotropic fireballs with $1 \ll \gamma \ll 100$, which cannot successfully generate $\gamma$-ray bursts but do can produce afterglows. Anyway, a successful application of the method would involve two important ingredients: the orphan needs to be discovered as early as possible and needs to be followed as long as possible. In fact, it has been suggested that radio searches, which are capable of finding potential GRB remnants as old as hundreds of days, may provide the best hope to find the missing orphans (Dalal et al. 2002; Totani \& Panaitescu 2002; Levinson et al. 2002). Again we see that theoretical investigation of GRB afterglows in the deep Newtonian phase is essential.

\section{ACKNOWLEDGMENTS}

We thank an anonymous referee for valuable comments and suggestions. YFH also thanks Y. Lu and J. C. S. Pun for stimulating discussion. This research was supported by a RGC grant of Hong Kong SAR, the National Natural Science Foundation of China, the Special Funds for Major State Basic Research Projects, and the Foundation for the Author of National Excellent Doctoral Dissertation of P. R. China (Project No: 200125).

\section{REFERENCES}

Akerlof C. et al., 1999, Nat, 398, 400

Berger E. et al., 2000, ApJ, 545, 56

Biretta J. A., Sparks W. B., Macchetto F., 1999, ApJ, 520, 621

Castro-Tirado A. et al., 1999, Sci, 283, 2069

Castro-Tirado A. et al., 2001, A\&A, 370, 398

Cheng K. S., Huang Y. F., Lu T., 2001, MNRAS, 325, 599

Chevalier R. A., Li Z.-Y., 2000, ApJ, 536, 195

Costa E. et al., 1997, Nat, 387, 783

Dado S., Dar A., De Rújula A., 2002, A\&A, 388, 1079

Dai Z. G., Lu T., 1998, MNRAS, 298, 87

Dai Z. G., Lu T., 1999, ApJ, 519, L155

Dai Z. G., Lu T., 2000, ApJ, 537, 803

Dai Z. G., Huang Y. F., Lu T., 1999, ApJ, 520, 634

Dalal N., Griest K., Pruet J., 2002, ApJ, 564, 209

Dar A., 1998, ApJ, 500, L93

Dermer C. D., Böttcher M., Chiang J., 1999a, ApJ, 515, L49

Dermer C. D., Chiang J., Böttcher M., 1999b, ApJ, 513, 656

Dermer C. D., Humi M., 2001, ApJ, 556, 479

Frail D. A. et al., 2001, ApJ, 562, L55

Frail D. A., Kulkarni S. R., Nicastro S. R., Feroci M., Taylor G. B., 1997, Nat, 389, 261

Frail D. A., Waxman E., Kulkarni S. R., 2000, ApJ, 537, 191

Fruchter A. S. et al., 1999, ApJ, 516, 683

Fruchter A. S. et al., 2000, ApJ, 545, 664

Galama T. J. et al., 1997, Nat, 387, 479

Galama T. J. et al., 1998a, ApJ, 500, L101

Galama T. J., Wijers R. A. M. J., Bremer M., Groot P. J., Strom R. G., Kouveliotou C., van Paradijs J., 1998b, ApJ, 500, L97

Garcia M. R. et al., 1998, ApJ, 500, L105

Granot J., Panaitescu A., Kumar P., Woosley S. E., 2002, ApJ, 570, L61

Harrison F. A. et al., 1999, ApJ, 523, L121

Hjorth J. et al., 2002, ApJ, 576, 113

Holland S., Bjornsson G., Hjorth J., Thomsen B., 2000, A\&A, 364,467

Huang Y. F., Dai Z. G., Wei D. M., Lu T., 1998a, MNRAS, 298, 459

Huang Y. F., Dai Z. G., Lu T., 1998b, A\&A, 336, L69

Huang Y. F., Dai Z. G., Lu T., 1999a, Chin. Phys. Lett., 16, 775

Huang Y. F., Dai Z. G., Lu T., 1999b, MNRAS, 309, 513

Huang Y. F., Gou L. J., Dai Z. G., Lu T., 2000a, ApJ, 543, 90

Huang Y. F., Dai Z. G., Lu T., 2000b, MNRAS, 316, 943

Huang Y. F., Dai Z. G., Lu T., 2000c, A\&A, 355, L43

Huang Y. F., Dai Z. G., Lu T., 2002, MNRAS, 332, 735

Ioka K., Nakamura T., 2001, ApJ, 561, 703

Jensen B. L. et al., 2001, A\&A, 370, 909

Katz J., 1994, ApJ, 422, 248

Kulkarni S. R. et al., 1998, Nat, 393, 35

Kulkarni S. R. et al., 1999, Nat, 398, 389

Kulkarni S. R., 2002, IAU Symposium No. 214 (Suzhou, China), to appear in the proceedings, eds. X. D. Li, Z. R. Wang, V. Trimble

Kobayashi S., Piran T., Sari R., 1999, ApJ, 513, 669 
Königl A., Granot J., 2002, ApJ, 574, 134

Levinson A., Ofek E., Waxman E., Gal-Yam A., 2002, ApJ, 576 , 923

Livio M., Waxman E., 2000, ApJ, 538, 187

Masetti N. et al., 2000, A\&A, 359, L23

Mészáros P., 2002, ARA\&A, 40, 137

Mészáros P., Laguna P., Rees M. J., 1993, ApJ, 415, 181

Mészáros P., Rees M. J., 1992, MNRAS, 257, 29P

Mészáros P., Rees M. J., 1997, ApJ, 476, 232

Mészáros P., Rees M. J., 1999, MNRAS, 306, L39

Mészáros P., Rees M. J., Papathanassiou H., 1994, ApJ, 432, 181

Mészáros P., Rees M. J., Wijers R. A. M. J., 1998, ApJ, 499, 301

Mészáros P., Rees M. J., Wijers R. A. M. J., 1999, New Astronomy, 4, 303

Metzger M., Djorgovski S. G., Kulkarni S. R., Steidel C. C., Adelberger K. L., Frail D. A., Costa E., Frontera F., 1997, Nat, 387,878

Moderski R., Sikora M., Bulik T., 2000, ApJ, 529, 151

Nakar E., Piran T., 2003, New Astron, 8, 141

Nakar E., Piran T., Granot J., 2002, ApJ, 579, 699

Panaitescu A., Kumar P., ApJ, 2001a, 554, 667

Panaitescu A., Kumar P., ApJ, 2001b, 560, L49

Panaitescu A., Kumar P., ApJ, 2002, 571, 779

Panaitescu A., Mészáros P., 1998, ApJ, 493, L31

Panaitescu A., Mészáros P., 1999, ApJ, 526, 707

Pedersen H. et al., 1998, ApJ, 496, 311

Perley R. A., Bridle A. H., Willis A. G., 1984, ApJS, 54, 291

Piran T., 1999, Phys. Report, 314, 575

Piro L. et al., 1998, A\&A, 331, L41

Ramirez-Ruiz E., Lloyd-Ronning N. M., 2002, New Astronomy, 7,197

Rees M. J., Mészáros P., 1992, MNRAS, 258, 41P

Rees M. J., Mészáros P., 1994, ApJ, 430, L93

Rhoads J., 1997, ApJ, 487, L1

Rhoads J., 1999, ApJ, 525, 737

Sari R., 1998, ApJ, 494, L49

Sari R., Narayan R., Piran T., 1996, ApJ, 473, 204

Sari R., Piran T., Halpern J. P., 1999, ApJ, 519, L17

Sari R., Piran T., Narayan R., 1998, ApJ, 497, L17

Shaviv N. J., Dar A., 1995, ApJ, 447, 863

Totani T., 1999, MNRAS, 307, L41

Totani T., Panaitescu A., 2002, ApJ, 576, 120

van Paradijs J., Kouveliotou C., Wijers R. A. M. J., 2000, ARA\&A, 38, 379

Vietri M., 1997, ApJ, 488, L105

Vreeswijk P. M. et al., 1999, ApJ, 523, 171

Waxman E., 1997a, ApJ, 485, L5

Waxman E., 1997b, ApJ, 489, L33

Waxman E., 1997c, ApJ, 491, L19

Weiler K. W., Panagia N., Montes M. J., Sramek R. A., 2002, ARA\&A, 40, 387

Wijers R. A. M. J., Galama T. J., 1999, ApJ, 523, 177

Wijers R. A. M. J., Rees M. J., Mészáros P., 1997, MNRAS, 288, L51

Wijers R. A. M. J. et al., 1999, ApJ, 523, 33

Yamazaki R., Ioka K., Nakamura T., 2002, ApJ, 571, L31

Zhu J. et al., 1999, IAU Circ. 7095
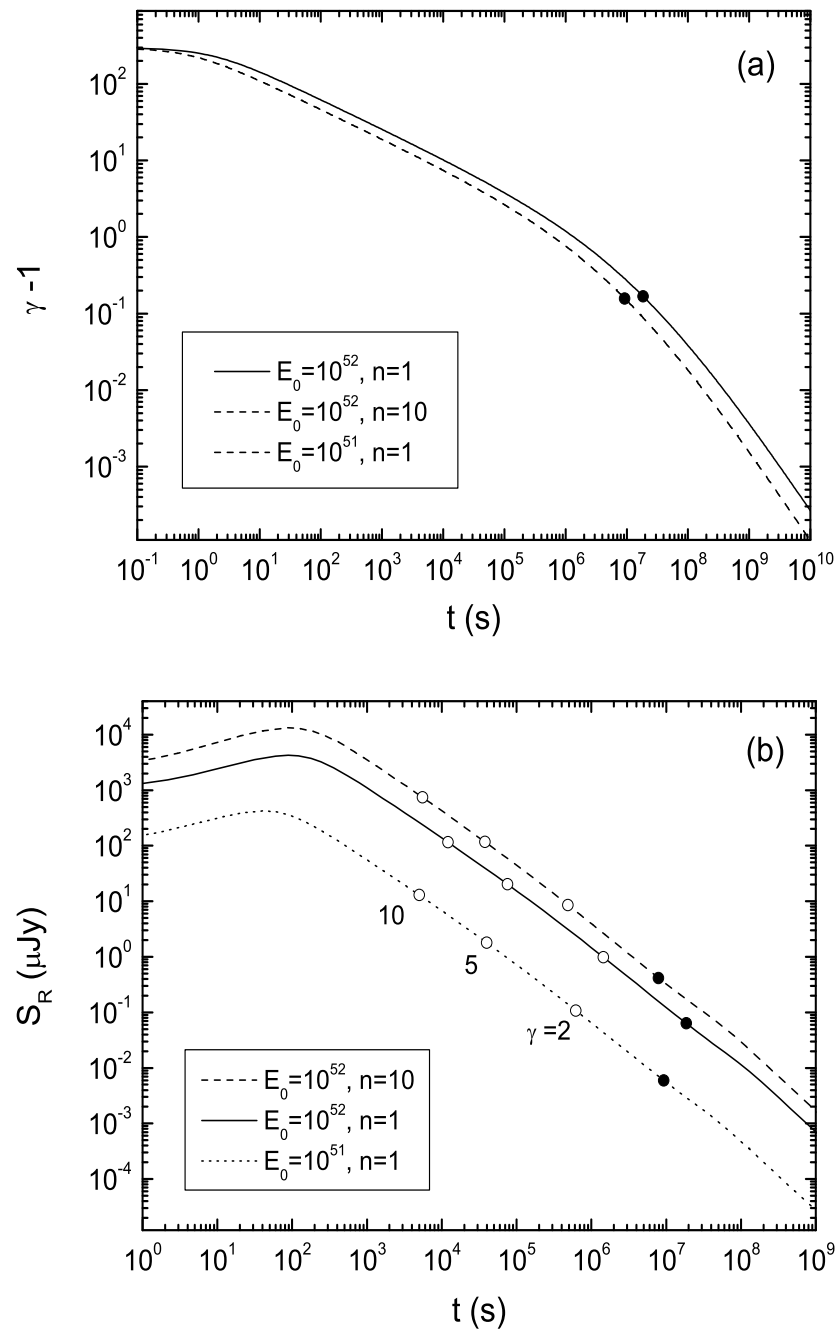

Figure 1. Evolution of the Lorentz factor for isotropic fireballs (a) and the corresponding $R$-band optical afterglows (b). We take $\xi_{\mathrm{B}}^{2}=10^{-4} . E_{0}$ and $n$ values are marked in units of erg and $\mathrm{cm}^{-3}$ respectively. Other parameters are evaluated in the first paragraph of Section 3. The black dot on each curve indicates the moment when $\gamma_{\mathrm{e}, \mathrm{min}}=\gamma_{\mathrm{e}, \mathrm{syn}}$, and the open circles on the light curves mark the time when $\gamma=2,5$ and 10 respectively. Note that the evolution of $\gamma$ is almost identical for the cases of $E_{0}=10^{52} \mathrm{erg}, n=10 \mathrm{~cm}^{-3}$ and $E_{0}=10^{51} \mathrm{erg}, n=1 \mathrm{~cm}^{-3}$. 

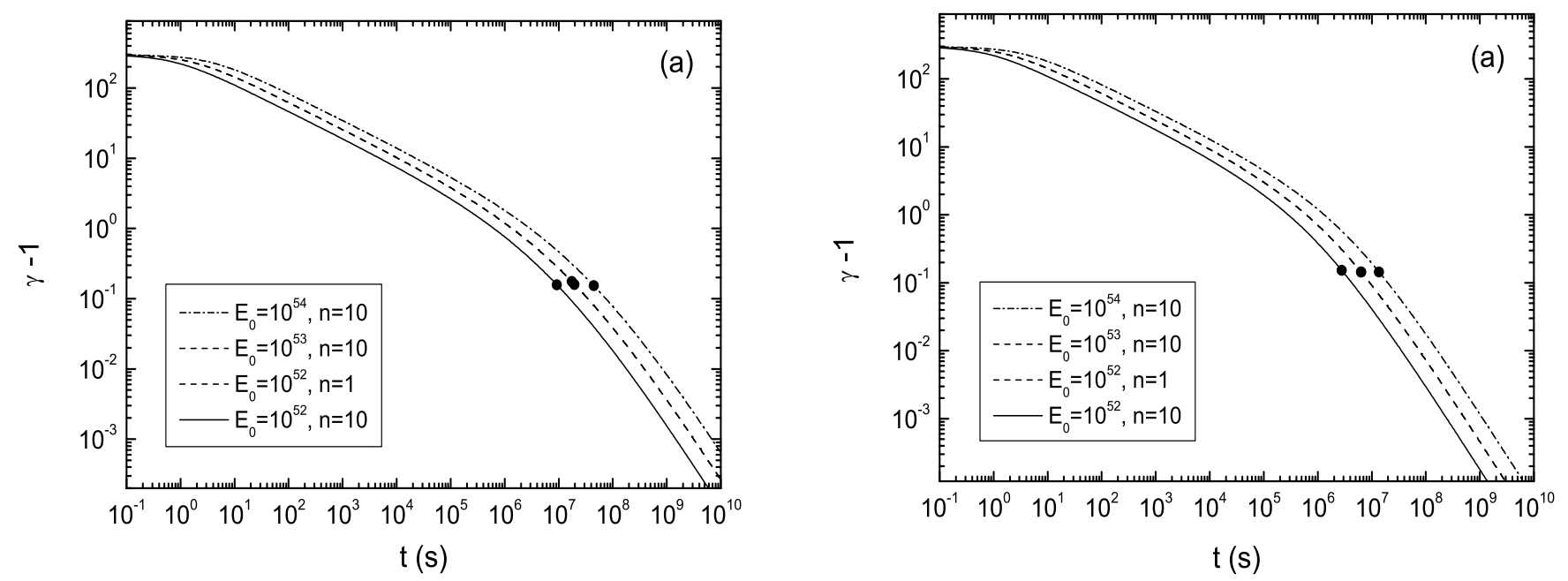

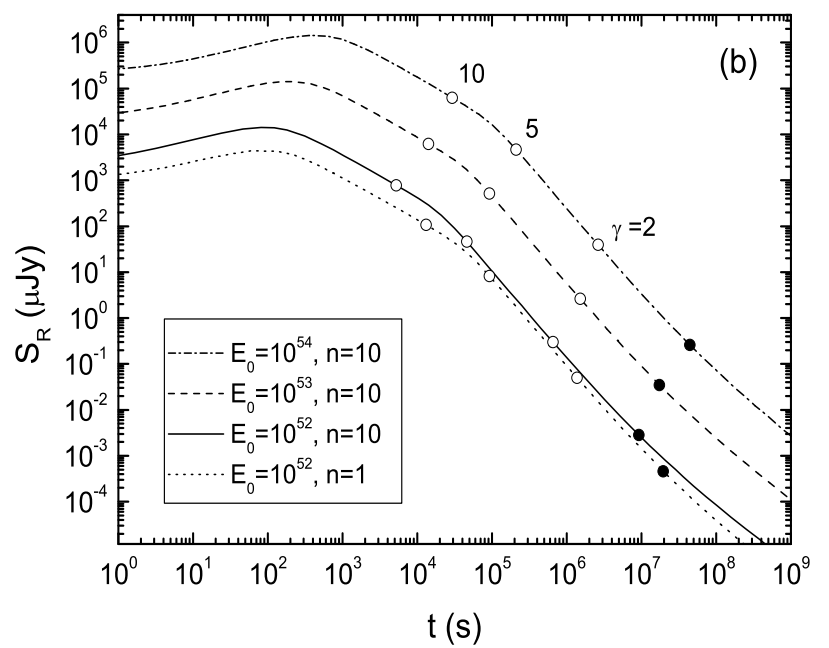

Figure 2. Evolution of the Lorentz factor for conical jets without lateral expansion (a) and the corresponding optical afterglows (b). We take $\theta_{0}=0.1, \xi_{\mathrm{B}}^{2}=10^{-4}$. Isotropic equivalent energy $E_{0}$ and $n$ values are marked in units of erg and $\mathrm{cm}^{-3}$ respectively. Other parameters are evaluated in the first paragraph of Section 3. Observers are assumed to be on the axis of the jet. The black dots and the open circles have the same meaning as in Fig. 1. Note that the evolution of $\gamma$ is almost identical for the cases of $E_{0}=10^{53} \mathrm{erg}, n=10 \mathrm{~cm}^{-3}$ and $E_{0}=10^{52} \mathrm{erg}, n=1 \mathrm{~cm}^{-3}$.

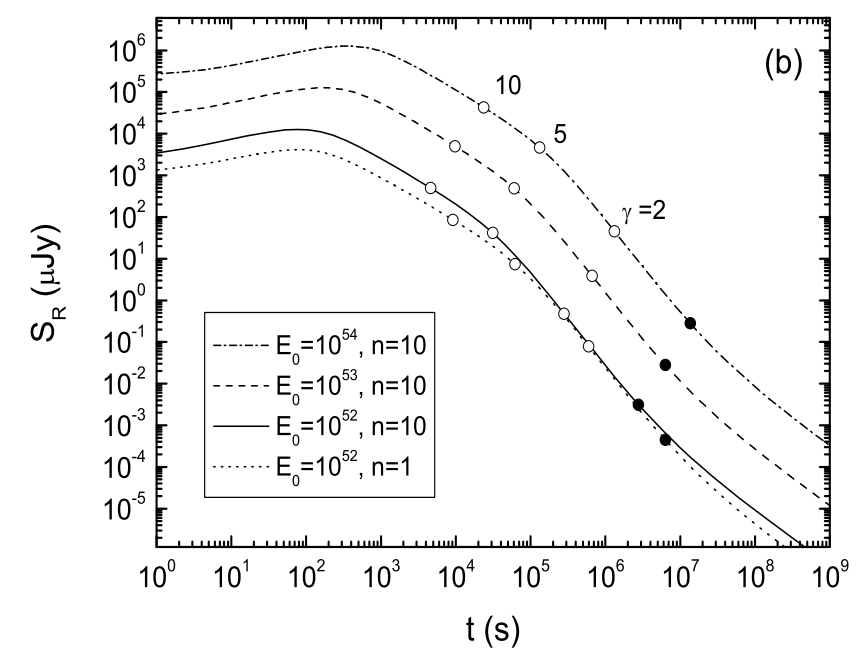

Figure 3. Same as Fig. 2 except that the conical jets here are expanding laterally at comoving sound speed. 

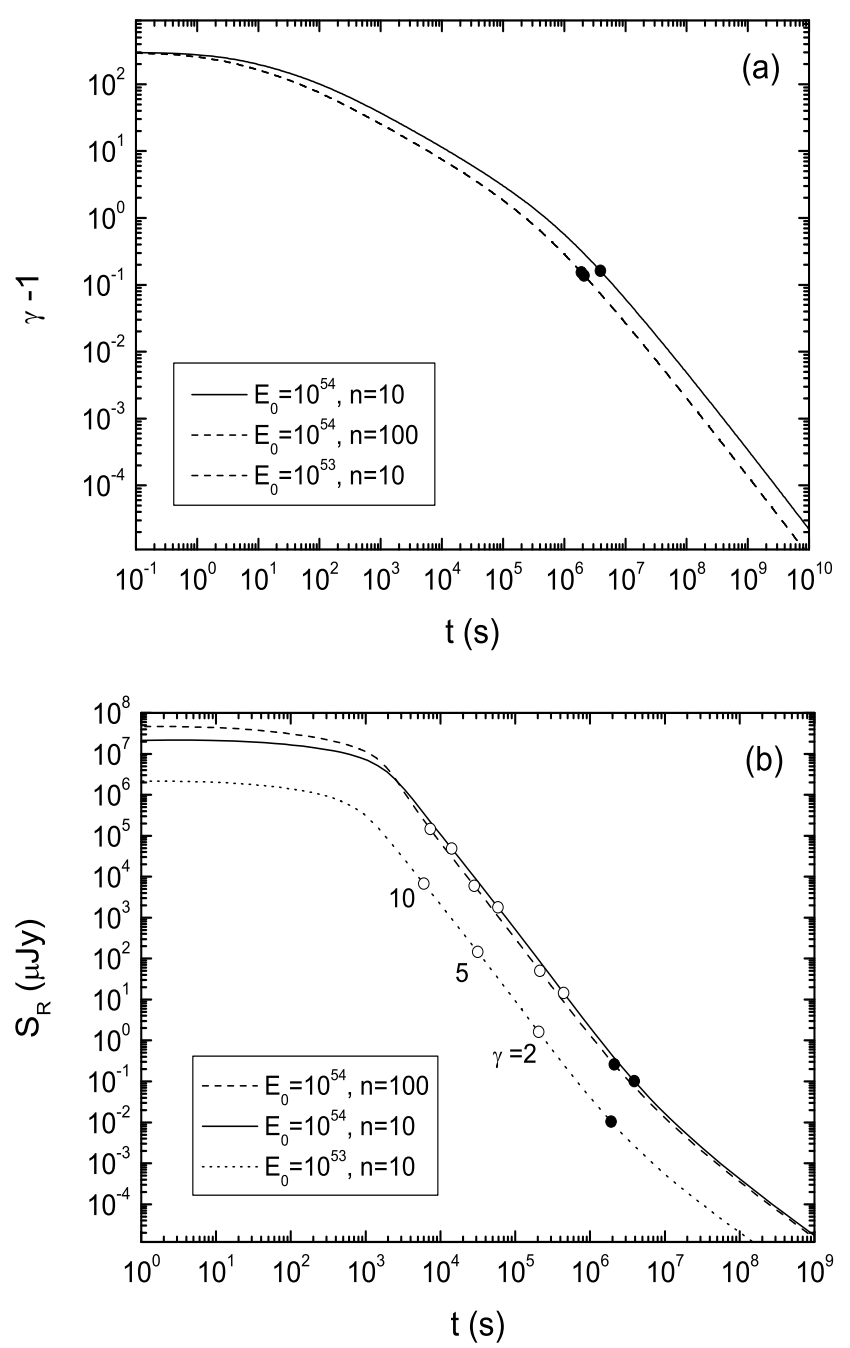

Figure 4. Evolution of the Lorentz factor for cylindrical jets with lateral expansion (a) and the corresponding optical afterglows (b). We take $\xi_{\mathrm{B}}^{2}=0.01$ and assume that the initial comoving lateral radius of the jet is $a_{0}=0.01 R_{0}$. Isotropic equivalent energy $E_{0}$ and number density $n$ are marked in units of erg and $\mathrm{cm}^{-3}$ respectively. Other parameters are evaluated in the first paragraph of Section 3. Observers are assumed to be on the axis of the jet. The black dots and the open circles have the same meaning as in Fig. 1. Note that the evolution of $\gamma$ is almost identical for the cases of $E_{0}=10^{54} \mathrm{erg}, n=100 \mathrm{~cm}^{-3}$ and $E_{0}=10^{53} \mathrm{erg}, n=10$ $\mathrm{cm}^{-3}$. 